\title{
Intervenções psicoeducativas dirigidas a familiares de indivíduos com transtorno bipolar
}

\section{Psychoeducational interventions for relatives of individuals with bipolar disorder}

\author{
Joana Cabral*; Célia Barreto Carvalho*; Paula Castilho Freitas**; Carlos Pato*** \\ *Universidade dos Açores; ** Universidade de Coimbra; *** College of Medicine Suny
}

\begin{abstract}
Resumo
Os familiares/cuidadores informais (CI) de indivíduos com transtornos mentais graves, como é o caso do Transtorno Bipolar (TB), estão sujeitos a um conjunto de dificuldades. Deste modo, é internacionalmente reconhecia a necessidade de se consolidar o apoio prestado a este tipo de CI. O presente estudo tem como objetivo analisar as características de intervenções destinadas a CI de indivíduos com TB, a partir de uma revisão sistemática da literatura. Os artigos analisados destacam que estas intervenções se revelam eficazes na redução da sobrecarga do CI e na melhoria da sua capacidade de cuidar, o que, por sua vez, se traduz na redução de recaídas do indivíduo com TB.

Palavras-chave: Doente, Família, Psicoeducação, Intervenção.

\section{Abstract}

The Relatives/ Informal Caregivers (IC) of individuals with severe mental disorders, such as Bipolar Disorder (TB), are subject to a number of difficulties.Therefore, it is internationally recognized the need to consolidate the support given to this type of IC. The present study is a review of the literature aims to analyze the characteristics of interventions for IC of individuals with TB. The papers analyzed highlight that these interventions prove to be effective in reducing IC overload and improving their ability to care, which, translates into reducing relapses of the individual with TB.

Keywords: Patient, Family, Psychoeducation, Intervention.
\end{abstract}

Atualmente, é reconhecida a importância dos cuidadores informais (CI) de indivíduos com Transtornos de Humor crónicos (Kumar \& Gupta, 2014), os quais poderão ser familiares, amigos ou vizinhos, que prestam suporte ao doente a vários níveis, em contexto não hospitalar/comunitário, sem serem compensados/remunerados por isso (Bastawrous, 2013). A experiência de cuidar informalmente de um doente com TB, implica uma dedicação e esforço constante que, por si só, pode ser extremamente desgastante e exigente (World federation for mental health (WFMH), 2010), especialmente se os familiares/cuidadores não receberem o devido suporte psicossocial (Fiorillo et al., 2014).

Internacionalmente, têm sido desenvolvidos programas de intervenção psicossociais dirigidos a CI de doentes psiquiátricos, sendo que a abordagem psicoeducativa e os princípios do modelo cognitivo-comportamental, não só estão presentes na maioria das intervenções, como, são recomendados, sobretudo pelos resultados positivos atingidos, e que se reportam ao aumento da resiliência e qualidade de vida do CI, bem como à diminuição da taxa de recaídas dos doentes beneficiários (Levy-Frank, Hasson-Ohayon, Kravetz, \& Roe, 2011; Mirabel-Sarron et al., 2015; Reinares et al., 2016). Por conseguinte, torna-se necessário analisar as características e eficácia das escassas intervenções desenvolvidas e testadas para apoiar especificamente os cuidadores informais de indivíduos com $\mathrm{TB}$, visto que é a partir da exploração do conhecimento disponível sobre as mesmas que se poderá ponderar a sua replicação ou o desenvolvimento de novas intervenções neste âmbito, no sentido de se oferecer um suporte mais consistente a estes cuidadores.

\section{Psicoeducação}

A abordagem interventiva mais utilizada em programas psicossociais destinados a apoiar CI de indivíduos com TB é a psicoeducação, a qual tem vindo a ser amplamente ministrada, quer ao doente, quer aos seus CI, por se reconhecer os benefícios que esta pode trazer para ambas as partes (Justo, Soares, \& Calil, 2007; Reinares et al., 2016). A psicoeducação é um meio complementar ao tratamento de doenças psiquiátricas graves, nomeadamente a bipolaridade, revelando-se eficaz na melhoria do estado psicológico dos participantes e na redução de recaídas dos doentes (McFarlane, Dixon, Lukens, \& Lucksted, 2003).

A abordagem psicoeducativa rege-se pelos princípios teóricos da Terapia Cognitivo-Comportamental (Mirabel-Sarron et al., 2015), caracterizando-se por ser estruturada, diretiva, limitada no tempo, focada no presente e no desenvolvimento de competências (McFarlane et al, 2003; Pitschel-Walz, Rummel-Kluge, 
Reichhart, Bäuml, \& Kissling, 2007; Reichhart, Pitschel-walz, Kissling, \& Bäuml, 2010). A psicoeducação familiar tem como principais objetivos fornecer às famílias a informação que necessitam sobre o problema psiquiátrico da pessoa de quem cuidam e disponibilizar-lhes estratégias de coping adequadas para lidar com as dificuldades associadas ao processo de cuidar de indivíduos com problemas desta natureza (Lefley, 2009).

Entre as intervenções direcionadas a indivíduos com TB e aos seus familiares/ CI que incluem uma abordagem psicoeducativa, a Family-Focused Therapy (FTT) é a mais popular, o que se deve tanto à forma rigorosa com que foi desenvolvida e testada, como à consistência dos resultados que demostram a sua eficácia a vários níveis (Fiorillo et al., 2013; Justo et al., 2007).

\section{Family-Focused Therapy (FTT)}

A FFT foi criada tendo como referência a estrutura do programa de Falloon, Boyd, e McGill (1984) desenvolvido originalmente para a esquizofrenia, tendo sido substancialmente adaptado por Miklowitz e Goldstein (1997) para os indivíduos com transtorno bipolar e suas famílias. Este programa é composto por 21 sessões de 1 hora cada, que se subdividem em três componentes principais: psicoeducação sobre o TB (informações sobre as características da doença, tratamento, sinais de alerta precoce e gestão de comportamentos suicidas); desenvolvimento de competências de comunicação e treino de resolução de problemas (Miklowitz et al., 2004).

Os resultados dos estudos de eficácia da TFF revelaram a sua capacidade de contribuir para reduzir o número de recaídas e aumentar o tempo entre crises dos indivíduos com TB (Miklowitz et al., 2000; Miklowitz, George, Richards, Simoneau, \& Suddath, 2003; Rea et al., 2003). Para além disso, demostrou possuir um maior efeito na redução de sintomas depressivos do que nos sintomas de hipomania e/ou mania (Miklowitz et al., 2000, 2003). No entanto, apesar de parecer reduzir a carga subjetiva e a emoção expressa dos CI (Miklowitz et al, 2009) o impacto que a TFF produz nos famíliares/CI foi pouco explorado, havendo a necessidade de ser considerado em estudos futuros.

Apesar da FFT ter sido originalmente criada para ser aplicada simultaneamente a indivíduos com TB e seus familiares, Perlik (et al., 2011) aplicaram-na apenas aos CI, visto que há autores (eg. Madigan et al., 2012; Perlick et al., 2011; Reinares et al., 2008) que defendem que os CI retiram um maior proveito deste tipo de intervenção quando os doentes não estão presentes nas sessões.

\section{Intervenções exclusivamente para CI}

Reinares (et al., 2008) foi um dos impulsionadores da aplicação deste tipo de intervenções apenas a CI, visto que em experiências anteriores com grupos de psicoeducação destinados a indivíduos com TB e seus familiares/ CI, este autor percebeu que os cuidadores que participavam nestas intervenções não colmatavam totalmente as suas necessidades específicas, pedindo mais informação sobre como lidar com a doença e suas consequências. Assim, Reinares (et al., 2008), partindo desta lacuna por ele identificada, realizou um estudo que visou analisar a eficácia deste tipo de intervenção quando aplicada apenas aos cuidadores, onde apontou como possíveis potencialidade desta nova modalidade de intervenção o facto dos cuidadores, ao não estarem na presença do doente, se expressarem mais abertamente sobre os seus sentimentos e dificuldades relativamente ao doente a quem prestam cuidados, bem como beneficiarem de um maior à vontade para levantar questões que provavelmente não seriam colocadas se o doentes estivesse presente (Reinares et al., 2008, p.112). Para além disso, considerou que os cuidadores, ao participarem em grupos constituídos por indivíduos com características e problemas comum, retirariam um maior proveito da intervenção, por a mesma facilitar: a aprendizagem interpares; a partilha e reforço de estratégias de coping adequadas; a redução do estigma e o alargamento da rede social dos participantes (Reinares et al., 2008, p.112).

As vantagens destas intervenções se aplicarem apenas a cuidadores, não se ficam por aqui, pois, segundo Perlick (et al., 2011), esta é uma forma dos familiares/CI aumentarem a sua capacidade de cuidar e gerir o stress a que estão sujeitos, mesmo quando os pacientes não estão disponíveis para receber este tipo de apoio. Contudo, Reinares (et al., 2008) considera que o recomendável para que os programas direcionados aos cuidadores produzam efeitos mais significativos, é o fato de, em algum momento da sua aplicação envolverem também os doentes, sugerindo que se reserve uma ou duas sessões destinadas aos cuidadores e respetivos doentes.

Deste modo, o presente trabalho, tem como objetivo realizar uma revisão sistemática da literatura sobre as intervenções psicossociais dirigidas apenas a cuidadores informais de indivíduos adultos com TB.

\section{Metodologia}

Realizou-se uma revisão sistemática da literatura que teve como intuito identificar as principais características das intervenções direcionadas a cuidadores informais de indivíduos com TB e os efeitos que estas produzem nos seus participantes. Neste sentido, e numa primeira fase, elaborou-se um protocolo onde se definiram os princípios metodológicos para este trabalho. Posto isto, efetuou-se uma pesquisa exaustiva de artigos científicos publicados, nos últimos 10 anos, em revistas internacionais da especialidade sobre a temática em análise, com recurso à plataforma online ScienceDirect Elsevier. Seguidamente, procedeu-se à seleção e à sistematização da informação obtida tendo por base o protocolo supracitado

Foram selecionados apenas artigos referentes a estudos de eficácia de intervenções psicossociais direcionadas a familiares/cuidadores informais de indivíduos com perturbações de humor. 


\section{Resultados}

Entre a bibliografia analisada, apenas 6 artigos respeitavam os critérios de seleção definidos para este estudo, no entanto, 2 destes artigos foram eliminados da análise por se referirem a intervenções breves (eg. Workshops (Eisner \& Johnson, 2008; Ruffolo, Nitzberg, \& Schoof, 2011). Assim, analisaram-se aprofundadamente 4 artigos (Jonsson \& Danielson, 2011; Madigan et al., 2012; Perlick et al., 2011; Reinares et al., 2008), cuja sua informação mais relevante se encontra sistematizada na tabela 1 .

\section{Tabela 1}

Síntese de dados dos artigos analisados sobre a eficácia de intervenções dirigidas a cuidadores informais de indivíduos com transtorno bipolar

\section{Principais características das intervenções analisadas}

Autor(es) do estudo: Reinares et al., 2008

\section{Amostra}

113 Cuidadores de indivíduos adultos com diagnóstico de Bipolaridade (83\%, TB Tipo I; BDI e 17\% TB Tipo II) em estado eutimico pelo menos há 3 meses. Grupo controlo (GC) $\mathrm{N}=56$; Grupo experimental (GE) N=57.

O programa foi aplicado por várias vezes a grupos de 10-12 cuidadores.

\section{Intervenção analisada}

\section{Psychoeducation group intervention (PGI)}

Intervenção destinada apenas a cuidadores que se foca na transmissão de conhecimentos sobre o TB e no desenvolvimento de competências de coping.

$\mathrm{N}^{\mathrm{o}}$ de sessões

12 sessões de 90 minutos

Duração:

12 meses

Profissionais que ministraram o programa:

Psicólogo (o mesmo nas várias aplicações do programa).

\section{Principais resultados}

A intervenção (PGI) revelou ser eficaz na prevenção de episódios de hipomania ou mania, visto que os doentes cujos cuidadores participaram do grupo de intervenção tiveram menos recaídas comparativamente aos do grupo controle. No entanto, este programa não se revelou eficaz na prevenção de episódios depressivos.

Não encontraram diferenças significativas na prevenção de episódios depressivos e na adesão à medicação entre o grupo controlo e experimental.

$$
\text { Autor(es) do estudo: Perlick et al., } 2011
$$

\section{Amostra:}

46 cuidadores de indivíduos adultos com diagnóstico de Bipolaridade (85\%, TB Tipo I e $15 \%$ TB Tipo II); $60 \%$ em estado grave. FFT-HPII N=25; $\mathrm{HE} \mathrm{N}=21$.

\section{Intervenção analisada 1}

\section{Family Focused Treatment- Health Promoting}

\section{Intervention (FFT-HPI)}

Intervenção cognitivo-comportamental desenhada para fornecer ao cuidador competências para gerir a doença do familiar, promover o auto-cuidado, bem como reduzir a tensão, depressão e comportamentos de risco para a saúde. Baseia-se na intervenção desenhada por Miklowitz (2008).
$\underline{\mathrm{N}^{\circ} \text { de sessões }}$

$12-15$ sessões (semanais) de 45 minutos

Duração:

4 meses

Profissionais que ministraram o programa:

Dois terapeutas com formação em FFT e em terapias cognitivo-comportamentais.

\section{Intervenção analisada 2}

\section{Health Education (HE)}

Abordagem didática destinada a informar os cuidadores sobre os problemas de saúde e sobre preocupações que foram identificadas na literatura como sendo comuns e/ou relevantes para os cuidadores.

No estudo de Perlick et al, 2011 para testarem a eficácia desta intervenção, apresentaram as informações através de oito vídeos de 20-25 minutos (um por sessão). Os vídeos foram apresentados por um investigador, que ia parando os vídeos em zonas chave para garantir que os participantes estavam a perceber, no entanto absteve-se de discutir o material ou a própria situação do cuidador.

$\mathrm{N}^{\mathrm{o}}$ de sessões

$$
\text { 8-12 sessões }
$$

Duração:

4 meses

Profisssional que ministrou o programa:

Investigador.

\section{Principais resultados:}

Os cuidadores que participaram FFT-HPI, apresentaram uma maior redução dos sintomas depressivos, nos comportamentos de risco para com a saúde e na sobrecarga, comparativamente aos cuidadores que receberam HE. Esta melhoria foi parcialmente mediada pela redução dos comportamentos de evitamento dos cuidadores.

Os doentes cujos seus cuidadores participaram no FFT-HPI também revelaram uma maior redução dos sintomas depressivos, resultados que foram parcialmente mediados pela redução da sintomatologia psiquiátrica dos cuidadores.

Autor(es) do estudo: Jonsson \& Danielson, 2011

\section{Amostra:}

34 indivíduos que têm um grau de parentesco e vivem com uma pessoa diagnosticada com TH. A intervenção foi aplicada por várias vezes a grupos de 6-10 cuidadores.

\section{Intervenção analisada}

\section{Educational Intervention (EI)}

Visa aumentar a capacidade de gerir o papel como familiar de uma pessoa com TB. No estudo de Jonsson e Danielson (2011) as sessões de EI foram compostas por uma fase inicial de introdução a um tema, sendo seguidas de um espaço de reflexão e debate, onde os participantes tiveram a oportunidade de partilhar com os restantes grupos as suas experiências. Durante as sessões foram treinadas, a partir de role-play, algumas estratégias para lidar com o doente e no final de cada sessão foram entregues aos participantes um resumo das temáticas trabalhadas. A intervenção foi baseada no modelo cognitivo de Aaron Beck (Beck et al.1979), sendo que a relação entre os pensamentos, sentimentos e comportamento foi destacada durante as sessões.

$\mathrm{N}^{0}$ de sessões

10 sessões (semanais) de 90 minutos

Duração:

Sem informação.

Profissionais que ministraram o programa: 
Dois enfermeiros da área da psiquiatria com formação em terapia cognitiva.

\section{Principais resultados:}

Os resultados deste estudo revelam que a intervenção educativa aplicada a um grupo de familiares que vivem com uma pessoa com transtorno bipolar permitiu aumentar a sua compreensão sobre a doença, reduzindo o stress causado pela mesma, bem como facilitando o seu funcionamento social.

\section{Madigan et al., 2012}

Amostra:

47 Familiares cuidadores de 34 indivíduos com TB. MFGP N=18; SFGT N=19; TAU N=10.

\section{Intervenção analisada 1}

\section{Multifamily Group Psychoeducation (MFGP)}

A intervenção foi baseada na estrutura desenvolvida por Mueuser e colaboradores (1994) e adaptada para o TB de acordo com as diretrizes do programa de Miklowitz (2002) direcionado para cuidadores.

$\mathrm{N}^{\mathrm{o}}$ de sessões

5 sessões semanais de 2 horas cada

Duração:

5 semanas

Profissionais que ministraram o programa:

Um enfermeiro e um assistente social da área da psiquiatria.

\section{Intervenção analisada 2:}

\section{Solution Focussed Group Therapy (SFGT)}

Intervenção focada nas soluções e não nos problemas, isto é procura explorar os recursos atuais e esperanças futuras, em vez de apresentar problemas e causas passadas inspirada na obra de Miller e colaboradores (1996).

$\underline{\mathrm{N}^{\mathrm{o}} \text { de sessões }}$

5 sessões semanais

Duração:

5 semanas

Profisssional que ministraram o programa:

Dois enfermeiros da área da psiquiatria.

Intervenção analisada 3:

\section{Treatment as Usual (TAU)}

Apoio habitual sem uma intervenção especializada adicional, neste estudo assumiu o papel de Intervenção Controlo.

\section{Principais resultados:}

Os cuidadores que foram alvo de intervenção participando no programa MFGP ou no SFGP, revelaram ter melhorado significativamente o seu conhecimento sobre a patologia e apresentaram uma redução na sobrecarga geral e sofrimento psíquico no $1^{\circ}$ e $2^{\circ}$ anos de follow-up. Os referidos benefícios não foram observados no grupo controlo (TAU).

\section{Discussão}

As intervenções apresentadas nos quatro estudos analisados (Jonsson \& Danielson, 2011; Madigan et al., 2012; Perlick et al., 2011; Reinares et al., 2008), embora com dominações e características distintas (Psychoeducation group intervention (PGI); Family Focused Treatment- Health Promoting Intervention (FFT-HPI); Educational Intervention e Multifamily Group Psychoeducation (MFGP); Solution Focussed
Group Therapy (SFGT)) apresentam abordagens interventivas comuns que se coadunam com os princípios da psicoeducação e do modelo teórico da TCC. No geral, têm como objetivo principal transmitir informação relevante sobre o TB e desenvolver um conjunto de competências que beneficiam o processo de cuidar.

\section{Principais características das intervenções}

Para atingirem os objetivos a que se propõem, estes programas aplicam entre 10 a 18 sessões de 90 minutos cada (Jonsson \& Danielson, 2011; Perlick et al., 2011; Reinares et al., 2008) (com exceção do MFGP composto apenas por 5 sessões de 2 horas (Madigan et al., 2012)), nas quais aplicam diversas métodos de intervenção nomeadamente, o debate interpares e o role-play para treino de competências de comunicação ou de resolução de problemas (Jonsson \& Danielson, 2011). Para além disso, evidencia-se a presença do modelo cognitivo-comportamental (Beck, 2013) durante a aplicação dos programa ao, por exemplo, procurarem continuamente realçar a relação entre os pensamentos, sentimentos e comportamentos; trabalharem as crenças dos participantes em relação à doença ou reforçarem comportamentos (Jonsson \& Danielson, 2011; Perlick et al., 2011).

\section{Eficácia das intervenções}

De acordo com os principais resultados encontrados nos estudos sobre os programas em análise, estes revelam ser eficazes a aumentar o conhecimento dos participantes sobre a doença (Jonsson \& Danielson, 2011; Madigan et al., 2012); a reduzir a sobrecarga subjetiva proveniente da prestação de cuidados (Jonsson \& Danielson, 2011; Madigan et al., 2012; Perlick et al., 2011), bem como a mediar a redução dos sintomas depressivos (Perlick et al., 2011) e prevenir a ocorrência de episódios de hipomania e/ou mania (Reinares et al., 2008) dos indivíduos com TB cujos familiares participaram no programa. Estes resultados são ótimos indicadores das potencialidades deste tipo de intervenções, reforçando a relevância das mesmas.

\section{Pontos em que as intervenções divergem}

Apesar das intervenções analisadas, de uma forma ou de outra, alcançarem resultados positivos, estas possuem vários fatores que as distinguem, tais como: o estado de saúde dos doentes cuidados pelos participantes (por exemplo em fase eutimica ou em crise); a informação transmitida; o fornecimento de materiais complementares; a formação dos profissionais que as aplicam; a duração dos programas; as metodologias adotadas e o local onde as intervenções são ministradas. Para além disso, os estudos em análise adotaram diferentes desenhos e metodologias de investigação (por exemplo: $\mathrm{N}$ da amostra; instrumentos de avaliação utilizados; uso de grupo experimental e controlo; avaliarem o impacto do programa nos familiares e/ou doentes) o que se traduz numa limitação para o presente estudo, visto que condiciona a capacidade de se tecerem considerações consistentes sobre os seus resultados, 
bem como de se destacar uma intervenção como sendo mais recomendável.

\section{Conclusões}

O presente estudo é um indicador que este tipo de intervenções, embora se revelem vantajosas para os seus beneficiários, ainda são escassas e inconsistentes, havendo a clara necessidade de se continuar a investir neste âmbito para que o apoio prestado aos indivíduos com TB e seus CI seja cada vez mais sólido e eficaz, no sentido de se reduzir o impacto negativo que esta patologia acarreta (Fiorillo et al., 2014; Justo et al., 2007; Reinares et al., 2016).

Apesar de ser premente a necessidade de se intervir junto dos CI de indivíduos com $\mathrm{TH}$, as respostas a este nível ainda são escassas, facto que se expressa inequivocamente no reduzido número de estudos científicos sobre intervenções direcionadas a estes CI. No que se refere às intervenções dirigidas exclusivamente a CI, a pesquisa exaustiva de literatura realizada no presente estudo, demonstra que embora estes estudos sejam escassos comportam um conjunto de benefícios para os CI que se traduzem na prevenção de recaídas dos doentes por eles cuidados.

Apesar dos estudos de eficácia das várias intervenções aqui apresentadas revelarem resultados promissores, continuam a existir várias questões que necessitam ser melhor exploradas, apontando para a relevância de se continuar a investir em estudos neste âmbito.

\section{Referências}

Bastawrous, M. (2013). Caregiver burden?A critical discussion. International Journal of Nursing Studies, 50(3), http://doi.org/10.1016/j.ijnurstu.2012.10.005

Beck A.T., Rush A.J., Shaw B.F., et al. (1979). Cognitive Therapy of Depression. New York: Guilford Press.

Beck, J. S. (2013). Terapia Cognitiva-Comportamental: teoria e prática. (2nd ed.). Porto Alegre: Artmed.

Caqueo-Urízar, A., Rus-Calafell, M., Urzúa, A., Escudero, J., \& Gutiérrez-Maldonado, J. (2015). The role of family therapy in the management of schizophrenia: challenges and solutions. Neuropsychiatric Disease and Treatment, 11, 145-51. http://doi.org/10.2147/NDT.S51331

Eisner, L. R., \& Johnson, S. L. (2008). An acceptance-based psychoeducation intervention to reduce expressed emotion in relatives of bipolar patients. Behavior Therapy, 39(4), 375-85. http://doi.org/10.1016/j.beth.2007.11.001

Falloon, I. R. H., Boyd, J. L., \& McGill, C. W. (1984). Family care of schizophrenia: A problem-solving approach to the treatment of mental illness. New York: Guilford Press.

Fiorillo, A., Vecchio, V., Luciano, M., Sampognaa, G., Rosa, C., \& Malangone, C. (2014). Efficacy of psychoeducational family intervention for bipolar I disorder: A controlled, multicentric, real-world study. Journal of Affective Disorders, 172C, 291-299. http://doi.org/10.1016/j.jad.2014.10.021
Jonsson, P., \& Danielson, E. (2011). Outcomes of an educational intervention for the family of a person with bipolar disorder: a 2-year follow-up study. Journal of Psychiatric and Mental Health Nursing, 18, 333-341. http://doi.org/10.1111/j.1365-2850.2010.01671.x

Justo, L., Soares, B. G. de O., \& Calil, H. (2007). Family interventions for bipolar disorder. Cochrane Database Syst. Rev., 17. http://doi.org/DOI: 10.1002/14651858.CD005167.pub2

Kumar, K., \& Gupta, M. (2014). Clinical and socio-demographic determinants of psychological health and burden in family caregivers of patients with unipolar depression. Asian journal of psychiatry, 9, 51-56.

Lefley, H. P. (2009). Family psychoeducation for serious mental illness. Oxford University Press.

Levy-Frank, I., Hasson-Ohayon, I., Kravetz, S., \& Roe, D. (2011). Family psychoeducation and therapeutic alliance focused interventions for parents of a daughter or son with a severe mental illness. Psychiatry Research, 189(2), 173-9. http://doi.org/10.1016/j.psychres.2011.02.012

Madigan, K. et al. (2012). A randomised controlled trial of carer-focussed multi-family group psychoeducation in bipolar disorder. European Psychiatry, 27(4), 281284. http://doi.org/10.1016/j.eurpsy.2010.12.008

McFarlane, W. R., Dixon, L., Lukens, E., \& Lucksted, A. (2003). Family psychoeducation and schizophrenia: a review of the literature. Journal of Marital and Family Therapy, 29(2), 223-245. http://doi.org/10.1111/j.1752-0606.2003.tb01202.x

Miklowitz, D. J., George, E. L., Axelson, D. A., Kim, E. Y., Birmaher, B., Schneck, C., ... Brent, D. A. (2004). Family-focused treatment for adolescents with bipolar disorder. http://doi.org/10.1016/j.jad.2004.05.020

Miklowitz, D. J., George, E. L., Richards, J. A., Simoneau, T. L., \& Suddath, R. L. (2003). A Randomized Study of Family-Focused Psychoeducation and Pharmacotherapy in the Outpatient Management of Bipolar Disorder, 60.

Miklowitz, D.J. (2002). The bipolar disorder survival guide: what you and your family need to know. London: Guilford.

Miklowitz, D.J. (2008). Bipolar Disorder: A Family-Focused Treatment Approach (2nd ed.). New York: The Guilford Press.

Miller S, Hubble M, Duncan B. (1996). Handbook of solution-focused brief therapy. San Francisco: Jossey-Bass Publishers.

Mirabel-Sarron, C., Docteur, A., Siobud-Dorocant, E., Dardennes, R., Gorwood, P., \& Rouillon, F. (2015). Psychoeducation and CBT. Annales Medico-Psychologiques, 173, 85-90. http://doi.org/10.1016/j.amp.2014.12.005

Mueser, K., Gingrich S \& Rosenthal C. (1994). Educational family therapy for schizophrenia: a new model for clinical service and research. Schizophren Res, 13, 99-107.

Perlick, A., Miklowitz, D., Lopez, N., Chou, J., Kalvin, C., Adzhiashvili, V., \& Aronson, A. (2011). Family-focused treatment for caregivers of patients 
with bipolar disorder. Bipolar Disord, 12(6), 627-637. http://doi.org/10.1111/j.1399-5618.2010.00852.x.Fam ily-focused

Petitjean, F. (2011). Les effets de la psychoe The therapeutical benefits of psychoeducation, 169, 184187. http://doi.org/10.1016/j.amp.2011.02.011

Reinares, M., Bonnín, C. M., Hidalgo-Mazzei, D., Sánchez-Moreno, J., Colom, F., \& Vieta, E. (2016). The role of family interventions in bipolar disorder: A systematic review. Clinical Psychology Review, 43, 47-57. http://doi.org/10.1016/j.cpr.2015.11.010

Reinares, M., Colom, F., Moreno-Sánchez, J., Torrente, C., Martinez-Aran, A., Goikolea, J., ... Vieta, E. (2008). Impact of caregiver group psychoeducation on the course and outcome of bipolar patients in remission: a randomized controlled trial. Bipolar Disorders, 10, 511-519.

Ruffolo, M., Nitzberg, L., \& Schoof, K. (2011). One-session family work- shops for bipolar disorder and depression. Psychiatr Serv, 62(3), 323.

World federation for mental health (WFMH). (2010). Caring for the caregiver: Why your mental health matters when you are caring for others.

\section{Agradecimientos}

Trabalho incluído no projeto de investigação Self-care to care, com a referência M3.1.a/F/083/2015, cofinanciado pelo Fundo Regional da Ciência e Tecnologia, através do Eixo Prioritário 10: "Ensino e Aprendizagem ao Longo da Vida", do programa AÇORES2020. 